\title{
Zur Kenntnis der Thymusnucleinsäuren.
}

(II. Mitteilung.) ${ }^{1}$ )

Von

H. Steudel.

(Aus dem physiologischen Institut zu Heidelberg.)

(Der Redaktion zugegangen am 7. Dezember 1904.)

Im Anschluß an den in meiner ersten Mitteilung beschriebenen Versuch, die bei der Spaltung der Thymusnucleinsäure mit siedenden Säuren entstehenden stickstoffhaltigen Bestandteile möglichst quantitativ zu bestimmen, sind weiterhin folgende Resultate gewonnen:

$100 \mathrm{~g}$ lufttrockenes nucleinsaures Kupfer von demselben Präparat, das zum ersten Versuch benutzt war, wurden mit $300 \mathrm{~g}$ konzentrierter $\mathrm{H}_{2} \mathrm{SO}_{4}$ und $600 \mathrm{~g}$ Wasser 14 Stunden lang am Rückflußkühler gekocht. Die auf $1 \mathrm{l}$ aufgefüllte Reaktionsflüssigkeit enthielt dann $6,244 \mathrm{~g} \mathrm{~N}$.

$5 \mathrm{ccm}$, nach Kjeldahl verascht, sättigen ab $22,3 \mathrm{ccm} \mathrm{n/10-0xalsäure}$ $=0,03122 \mathrm{~g} \mathrm{~N}$ oder $6,244 \mathrm{~g} \mathrm{~N}$ in $1000 \mathrm{ccm}$.

Ferner wurden $50 \mathrm{ccm}$ mit Barythydrat genau neutralisiert und mit überschüssigem Baryumkarbonat abdestilliert. Dann wurden

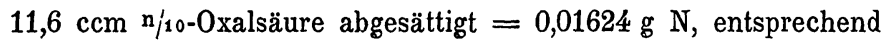
$0,3248 \mathrm{~g} \mathrm{~N}$ in $1000 \mathrm{ccm}$ als Ammoniakstickstoff.

Nach Entfernung der Schwefel- und Phosphorsäure mit Baryt wurde die wieder schwach mit Schwefelsäure angesäuerte Flüssigkeit noch einmal auf $1 \mathrm{l}$ aufgefüllt und der Gesamtstickstoff bestimmt.

$5 \mathrm{ccm}$ sättigen ab $19,4 \mathrm{ccm} \mathrm{n} / 10-$ Säure $=5,432 \mathrm{~g} \mathrm{~N}$ in $1000 \mathrm{ccm}$.

$5>,>19,5, \mathrm{n} / 10^{-},=5,460,>1000$,

1) Diese Zeitschrift, Bd. XLII, S. 165. 
Es waren also rund $0,4108 \mathrm{~g} \mathrm{~N}$ als sogenannter Huminstickstoff verloren.

Nun wurde die Flüssigkeit auf einen Gehalt von $5 \%$ Schwefelsäure gebracht und genau nach der in der ersten Mitteilung beschriebenen Methode weiter verarbeitet. Es ließen sich so isolieren

\begin{tabular}{|c|c|}
\hline Guanin & $=1,3552 \mathrm{~g}$ mit $0,6287 \mathrm{~g} \mathrm{~N}$ \\
\hline Adeninpikrat $=5,3526 \mathrm{~g}=1,975$ & $\mathrm{~g}$ Adenin $>1,0230>$ \\
\hline Cytosinpikrat $=5,8318,=1,886$ & , Cytosin > 0,7158 , , \\
\hline Thymin & $=3,69 \mathrm{~g}>0,8199$, , \\
\hline
\end{tabular}

Setzt man den Stickstoff in der Ausgangsmenge 6,244 $\mathrm{g}$ $=100$, so erhält man in Prozenten des Gesamtstickstoffs

$\begin{array}{ll}\mathrm{NH}_{8}-\text { Stickstoff } & =5,20 \% \\ \text { Humin- } & =6,58 \% \\ \text { Guanin- : } & =10,07 \% \\ \text { Adenin- : } & =16,39 \% \\ \text { Cytosin- : } & =11,47 \% \\ \text { Thymin- : } & =13,11 \%\end{array}$

Ferner wurden $100 \mathrm{~g}$ nucleinsaures Kupfer vom gleichen Präparat mit $300 \mathrm{ccm}$ konzentrierter Salzsäure vom spezifischen Gewicht 1,19, $200 \mathrm{~g}$ Wasser und $75 \mathrm{~g}$ Zinnchlorür 14 Stunden lang gekocht, dann in der auf 1000 aufgefüllten Reaktionsflüssigkeit Gesamtstickstoff und Ammoniakstickstoff bestimmt.

$5 \mathrm{ccm}$, nach Kjeldahl verbrannt, sättigen ab $24,5 \mathrm{ccm} \mathrm{n/10-Säure}$ $=6,846 \mathrm{~g} \mathrm{~N}$ in $1000 \mathrm{ccm}$.

$50 \mathrm{ccm}$ liefern, mit $\mathrm{BaCO}_{3}$ destilliert, $0,05502 \mathrm{~g} \mathrm{~N}$ als $\mathrm{NH}_{3}-\mathrm{N}$ $=1,1004 \mathrm{~g}$ in $1000 \mathrm{ccm}$.

Nachdem das Zinn mit Schwefelwasserstoff, die Salzsäure zum größten Teil durch wiederholtes Abrauchen auf dem Wasserbade, zum Schluß bei niederer Temperatur und unter Zugabe von Alkohol entfernt war, ließen sich aus der Reaktionsmasse isolieren:

Guanin: $0,4642 \mathrm{~g}$ mit $0,2154 \mathrm{~g} \mathrm{~N}$.

Adeninpikrat: $1,7042 \mathrm{~g}=0,6272 \mathrm{~g}$ Adenin * 0,3256 , *

Cytosinpikrat: 5,6620 > $=1,832$, Gytosin $>0,6950$ >

Thymin:

$3,67 \mathrm{~g}>0,8155$,

In Prozenten des Gesamtstickstoffs ausgedrückt, ergeben sich folgende Werte: 


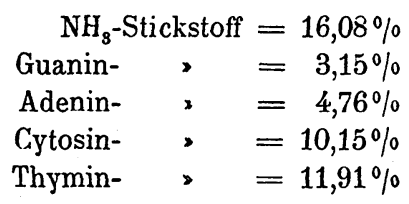

Den Huminstickstoff zu bestimmen, habe ich leider beim letzten Versuch versäumt; die Tabellen sind auch deshalb noch nicht ganz vollständig, weil die Alloxurbasenfraktion sowohl bei der Schwefelsäure- wie bei der Salzsäurespaltung einer quantitativen Bestimmung nach Abscheidung von Guanin und Adenin unerwartete Hindernisse in den Weg legte. Aber größere Änderungen wird die Aufarbeitung dieses Teiles, der übrigens nicht sehr bedeutend war, nicht mehr bringen, sodaß man wohl berechtigt ist, die entsprechenden Zahlen der drei Versuche miteinander $z u$ vergleichen. Ich stelle die drei Tabellen der Prozente des Gesamtstickstoffs hier nebeneinander:

\begin{tabular}{|c|c|c|c|}
\hline \multirow{2}{*}{$\begin{array}{l}\mathrm{N} \text { gefunden } \\
\text { in Form von }\end{array}$} & \multicolumn{3}{|c|}{ Spaltung mit: } \\
\hline & $\begin{array}{c}\text { Jodwasser- } \\
\text { stoffsäure }\end{array}$ & $\begin{array}{l}\text { Schwefel- } \\
\text { säure }\end{array}$ & $\begin{array}{l}\text { Salzsäure }+ \\
\text { Zinnchlorür }\end{array}$ \\
\hline $\mathrm{NH}_{8}-\mathrm{N}$. . . . . & 7,00 & 5,20 & 16,08 \\
\hline Humin-N . . & 11,54 & 6,58 & - \\
\hline Guanin-N . . . . & 3,61 & 10,07 & 3,15 \\
\hline Adenin-, & 13,45 & 16,39 & 4,76 \\
\hline Cytosin-s & 11,45 & 11,47 & 10,15 \\
\hline Thymin-s . . . & 15,88 & 13,11 & 11,91 \\
\hline
\end{tabular}

Ohne weiteres ergibt sich hieraus, daß die Schwefelsäurespaltung, die Kossel schon seit langer Zeit zur Aufteilung der Protamine und der Eiweißkörper benutzt, auch für die Untersuchung der Nucleinsäuren am geeignetsten ist. Bei ihr wird am wenigsten Ammoniak gebildet, nur etwas weniger wie bei der Jodwasserstoffspaltung, bedeutend weniger wie bei der Salzsäurespaltung.. Auch die Zahl für den Huminstickstoff ist bei der Schwefelsäurespaltung nur etwa halb so groß wie bei HJSpaltung. Dagegen sind die Zahlen für Guanin und Adenin bei der $\mathrm{H}_{2} \mathrm{SO}_{4}$-Spaltung am größten, wohl ein Beweis dafür, daß 
sie bei den beiden anderen Spaltungen mit zur Erhöhung des Humin-(HJ) resp. Ammoniakstickstoffs $(\mathrm{HCl})^{1}$ ) beigetragen haben. Die Werte für die einfachen Pyrimidinderivate zeigen in allen drei Versuchen gute Übereinstimmung, vom Cytosin wurde fast überall die gleiche Menge erhalten, vom Thymin desgleichen, nur konnte ich bei der $\mathrm{H}_{2} \mathrm{SO}_{4}$ - und $\mathrm{HCl}$-Spaltung keine nachweisbaren Mengen von Uracil auffinden. Daß trotz der starken Reduktionsmittel, die bei HJ- und HCl-Spaltung angewendet wurden, die Menge des Cytosins und Thymins nicht vermehrt worden ist, spricht sehr dafür, daß die einfachen Pyrimidinderivate unabhängig von den Alloxurkörpern aus dem Nucleinsäuremolekül hervorgehen.

Den Zahlen haften selbstverständlich alle Mängel eines ersten Versuches an, und bei Wiederholung der Spaltungen wird der eine oder andere Wert vielleicht noch um einige Einheiten korrigiert werden müssen, soviel aber ist bisher gewonnen, daß die Schwefelsäurespaltung zu wählen sein wird, wenn man eine Systematik der einzelnen Nucleinsäuren verschiedener Herkunft nach ihren Spaltungsprodukten versuchen oder die Mengenverhältnisse der einzelnen Basen in den $\mathrm{Ab}-$ bauprodukten der Nucleinsäuren feststellen will.

1) Adenin ist gegen Reduktionsmittel unbeständig. So geht es bei der Reduktion mit Zink und $\mathrm{HCl}$ schon bei Wasserbadtemperatur in einen Körper über, der vielleicht Azulminsäure ist. (Kossel, Diese Zeitschrift, Bd. XII, S. 248.) 